\title{
RELATIVE SCHUR MULTIPLIERS AND UNIVERSAL EXTENSIONS OF GROUP HOMOMORPHISMS
}

\author{
EMMANUEL D. FARJOUN YOAV SEGEV
}

\begin{abstract}
In this note, starting with any group homomorphism $f: \Gamma \rightarrow G$, which is surjective upon abelianization, we construct a universal central extension $u: U \rightarrow G$, under $\Gamma$ with the same surjective property, such that for any central extension $m: M \rightarrow G$, under $f$, there is a unique homomorphism $U \rightarrow M$ with the obvious commutation condition. The kernel of $u$ is the relative Schur multiplier group $H_{2}(G, \Gamma ; \mathbb{Z})$ as below. The case where $G$ is perfect corresponds to $\Gamma=1$. This yields homological obstructions to lifting solution of equations in $G$. Upon repetition, for finite groups, this gives a universal hypercentral factorization of the map $f: \Gamma \rightarrow G$.
\end{abstract}

\section{INTRODUCTION}

Let $G$ be a group. The purpose of this note is to extend the well known theorem saying that if $G$ is perfect, then there is a universal perfect central extension $\widehat{G} \rightarrow G$, whose kernel is the Schur multiplier $H_{2}(G ; \mathbb{Z})$ ( $\left.[\mathrm{Ka}]\right)$. We replace the condition of $G$ being perfect with the hypothesis that a map $\Gamma \rightarrow G$ induces a surjection on the abelian quotients.

Throughout this note we fix a group homomorphism $f: \Gamma \rightarrow G$, and we consider extensions

$$
0 \rightarrow K \rightarrow M \rightarrow G \rightarrow 1 .
$$

in which often $K=A$ is an abelian group. The following is the basic concept used in this note (compare with the end of section 4, p. 263 of Hochschild's paper $[\mathrm{H}]$.)

Definitions 1.1. (1) An f-extension of $G$ is a pair $(M, \psi)$, where $M$ is an extension of $G$ and $\psi: \Gamma \rightarrow M$ is a map-the structure map-that factorizes $f$ as in diagram (1.1) below. If the extension is central we refer to it as a central $f$-extension.

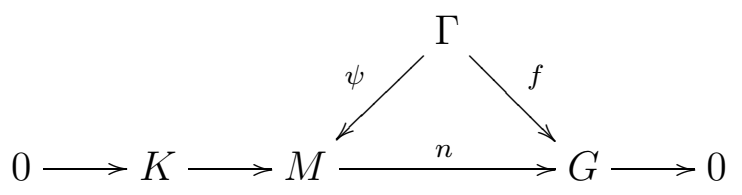

Date: September 17, 2021.

2010 Mathematics Subject Classification. Primary: 20E22 ,55U.

Key words and phrases. central extension, relative Schur multiplier, second homology, hypercenter, universal factorization. 
(2) A map between two $f$-extensions $(M, \psi)$ and $\left(M^{\prime}, \psi^{\prime}\right)$ of $G$ with kernels $K, K^{\prime}$ respectively, is a map of the underlying extensions which is the identity on $G$, as in the commutative diagram (2.1) below.

Notation and Remarks. Hochschild in his relative homological algebra paper deals with a similar situation for subgroups $\Gamma \subseteq G$, see $[\mathrm{H}$, section 3 and 4]. Our treatment here is for general group maps and this allows a simpler formulation. When there are no coefficients in sight, we take (co)homology with integral coefficients. Relative (co)homology groups are calculated with respect to our given fixed map $f$. That is, for an abelian group $A$, by $H_{*}(G, \Gamma ; A)$ we mean $H_{*}\left(B G \cup_{B f} \operatorname{Cone}(B \Gamma) ; A\right)$. A more careful-but still straightforwarddefinition of relative (co)-homology is needed for relative (co-)homology with local coefficients in a $G$-module $A$. The former is used to formulate the following classification statement which is a direct analogue of the classical absolute case $[\mathrm{H}]$ (see subsections 2.4, 2.5 below). We formulate it for central extensions but a similar result exists for a more general situation:

Proposition 1.2. The equivalence classes of central $f$-extensions of $G$, with a given abelian group $A$ as a kernel, have a natural abelian group structure (see Definition 2.1) and as such are classified by the relative cohomology group $H^{2}(G, \Gamma ; A)$, with coefficients $A$.

The following is an extension of the Schur universal extension to a relative case:

Theorem 1.3. Assume that the map $f_{a b}: \Gamma_{a b} \rightarrow G_{a b}$ induced on the abelianizations is surjective. Then there exists a universal central $f$-extension $(U, \eta)$ of $G$ with kernel $H_{2}(G, \Gamma)$, such that for any central $f$-extension $(E, \psi)$ of $G$, there is a unique map of central $f$-extensions (as in Definition 1.1(2)) from $U$ to $M$. Moreover the map $\eta_{a b}: \Gamma_{a b} \rightarrow U_{a b}$ is again surjective.

1.4. The universal hyper-central extension. In the absolute Schur case the universal central extension does not have non-split extensions, and its second homology group vanishes. In the present case the relative universal central extension has, in general, further non-split central $f$-extensions, and among them a universal one. However for finite groups this process of taking universal central extensions stops after a finite number of steps. In this way one gets a universal hypercentral extension with no further non-split $f$-central ones. By hyper central extension one simply means a composition $E_{n} \rightarrow E_{n-1} \rightarrow \cdots \rightarrow E_{1} \rightarrow E_{0}=G$ of a finite number of central extensions $E_{i+1} \rightarrow E_{i}$.

Thus one gets a tower of universal central $f$-extensions:

$$
\ldots \rightarrow U_{n} \rightarrow U_{n-1} \rightarrow \cdots U_{1}=U \rightarrow G .
$$

As mentioned above, for a map of finite groups this tower terminates with $U_{\infty}=U_{N}$ a finite group yielding a factorization $\Gamma \rightarrow U_{\infty} \rightarrow G$. This $U_{\infty}$ is the universal, both initial and terminal, factorization of the map $f$ in the following sense:

Theorem 1.5. Let $f: \Gamma \rightarrow G$ be a map of finite groups that induces surjection $f_{a b}: \Gamma_{a b} \rightarrow$ $G_{a b}$ on the abelianizations. Then $U_{\infty}=U_{N}$ for some natural $N>0$, and it is a universal hypercentral $f$-extension of $G$ : This extension maps uniquely to any other hypercentral $f$ extension of $G$. In addition it satisfies $H_{i}\left(U_{\infty}, \Gamma\right)=0$ for $i=1,2$ and it is terminal among 
all such factorizations under $f$ namely $f$-extensions $A \rightarrow E \rightarrow G$ with $H_{i}(E, \Gamma) \cong 0$ for $i=1,2$.

\section{2. $f$-CENTRAL EXTENSIONS AND RELATIVE SCHUR MULTIPLIER}

Recall that throughout this note

$$
f: \Gamma \rightarrow G
$$

is a fixed map. The following definitions are direct generalizations of the usual definitions of a group structure on extensions as in [M], the usual proofs extend here with only minor changes.

Definition 2.1. A map between to $f$-extensions $M$ and $M^{\prime}$ is a commutative diagram as below; these extensions are called equivalent if $\kappa$ below is the identity-and thus $\tau$ is an isomorphism:

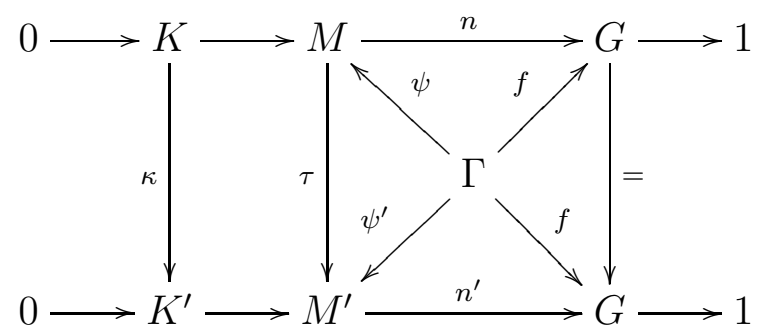

We denote by $\operatorname{Ext}^{\Gamma}(G, A)$ the set of all equivalence classes of central $f$-extensions of $G$ with kernel $A$ (where the map $f$ is suppressed from the notation). We now recall and extend the usual group structure on $\operatorname{Ext}^{\Gamma}(G, A)$. The additional information that we insert is the map from $\Gamma$ to the added extension. Let

$$
0 \rightarrow A \stackrel{\alpha}{\rightarrow} M \stackrel{\beta}{\rightarrow} G \rightarrow 1, \quad \psi
$$

and

$$
0 \rightarrow A \stackrel{\alpha^{\prime}}{\rightarrow} M^{\prime} \stackrel{\beta^{\prime}}{\rightarrow} G \rightarrow 1, \quad \psi^{\prime}
$$

be two $f$-extensions. Consider the following diagram

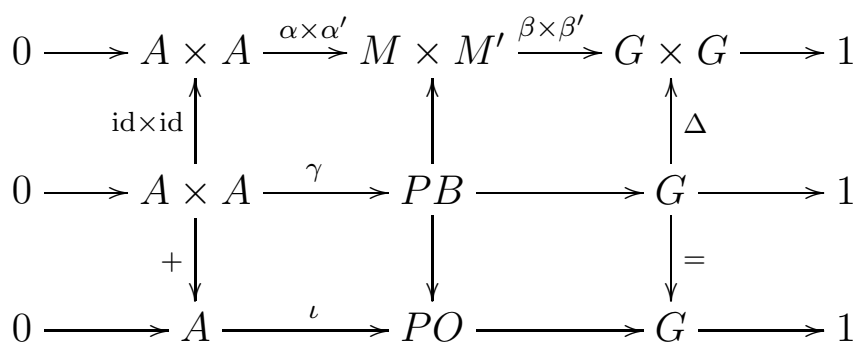


Here, $\Delta(g)=(g, g)$ is the diagonal map, $P B$ is the pullback in the right upper square, the map $A \times A \stackrel{+}{\rightarrow} A$ is addition, and $P O$ is the push-out in the left lower square. That push-out maps to $G$ by the universal property of push-outs. Notice that we have the map $\psi \times \psi^{\prime}: \Gamma \rightarrow M \times M^{\prime}$ and the map $f: \Gamma \rightarrow G$, and that $\left(\psi \circ \psi^{\prime}\right) \circ\left(\beta \times \beta^{\prime}\right)=f \circ \Delta$, so by the universal property of pullbacks there is a map $\Gamma \rightarrow P B$, and consequently a map $\psi^{\prime \prime}: \Gamma \rightarrow P O$. Thus $\left(P O, \psi^{\prime \prime}\right)$ is the sum of the given extensions. (Notice that since $\alpha \times \alpha^{\prime}$ is injective, so is $\gamma$ and consequently also $\iota$ ).

The inverse of an element is its push-out along $-1: A \rightarrow A$.

Examples 2.2. (1) Assume that $\Gamma=G$ and that $f$ is the identity map. Then any two $f$-extensions of $G$ with kernel $A$ are isomorphic. Indeed, if $(M, \psi)$ and $\left(M^{\prime}, \psi^{\prime}\right)$ are such $f$-extensions, then we may assume that $A$ is contained in both $M$ and $M^{\prime}$, and then $M=A \rtimes \psi(G)$, the semi direct product, and $M^{\prime}=A \rtimes \psi^{\prime}(G)$ and the map $1_{A} \times\left(\psi^{-1} \circ \psi^{\prime}\right)$ is an isomorphism of extensions.

(2) Assume that $G=\{1\}$. Then one has $M \cong A$ and there is only one equivalence class of central extensions of $G$ with kernel $A$. However, for any map $\psi: \Gamma \rightarrow A$ we get that $(M, \psi)$ is an $f$-extension of $G$, and note that distinct maps $\psi$ will give inequivalent central $f$-extensions hence $\operatorname{Ext}^{\Gamma}(1 ; A) \cong \operatorname{Hom}(\Gamma, A)$.

(3) The neutral element of $\operatorname{Ext}^{\Gamma}(G ; A)$ is a (semi-direct product) split extension $0 \rightarrow A \rightarrow A \rtimes G \rightarrow G \rightarrow 1$, coming from the action of $G$ on $A$, where $\psi_{0}: \Gamma \rightarrow A \rtimes G$ is the map $\psi_{0}(\gamma)=(0, f(\gamma))$, for all $\gamma \in \Gamma$.

(4) Note that if $f$ itself is a central extension of $G$, then it is itself a (universal) central $f$-extension as in the theorem above, with the identity on $\Gamma$ as the structure map.

Notice that

Lemma 2.3. Let $M$ be an extension of $G$ with abelian kernel $A$, and let $M^{\prime}$ be the pull-back of $M$ along $f$ as in diagram (2.2) below:

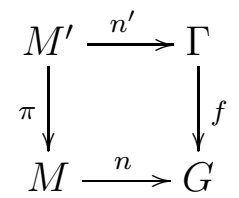

then

(1) if $M$ is central then $M^{\prime}$ is a central extension of $\Gamma$ with the same kernel $A$;

(2) if $(M, \psi)$ is an $f$-extension, then $M^{\prime}$ splits;

(3) conversely, if $n^{\prime}$ has a section $s: \Gamma \rightarrow M^{\prime}$, then $(M, \psi)$ is an $f$-extension, where $\psi=s \circ \pi: \Gamma \rightarrow M$.

Proof. Recall that $M^{\prime}=\{(m, \gamma) \mid m \in M, \gamma \in \Gamma$ and $n(m)=f(\gamma)\}$. Also $n^{\prime}$ and $\pi$ are the corresponding projection maps. Hence part (1) is clear.

(2): Consider the map $s: \Gamma \rightarrow M^{\prime}$ defined by $s(\gamma)=(\psi(\gamma), \gamma)$, for all $\gamma \in \Gamma$. Then $s \circ n^{\prime}=1_{\Gamma}$, is the identity on $\Gamma$, so $s$ is a section of $n^{\prime}$. 
(3): Clearly $\psi=s \circ \pi$ shows that $(M, \psi)$ is an $f$-extension of $G$.

2.4. Definition of relative homology and cohomology $H_{*}(G, \Gamma ; A)$ and $H^{*}(G, \Gamma ; A)$.

Given a map $f: \Gamma \rightarrow G$, and an abelian group $A$, we now consider the relative (co)homology of $f$ with coefficients in $A$. The obvious candidate obtained by taking classifying spaces works well, see below, but here is an algebraic exposition that follows the usual chain complex approach.

The (co)homology group is defined as the (co)homology of the (co)chain complex gotten as the mapping cone (compare [We, 1.5, p.18]). One starts with the usual chain map induced by the group map $\Gamma \rightarrow G$ :

$$
C_{*}(f): C_{*}(\Gamma ; \mathbb{Z}) \rightarrow C_{*}(G ; \mathbb{Z}) .
$$

Define $C_{*}(G, \Gamma ; \mathbb{Z})$ to be the mapping cone of $C_{*}(f)$ :

$$
C_{n}(G, \Gamma):=C_{n}(G) \oplus C_{n-1}(\Gamma) \quad \text { with } \quad d(x, y)=\left(d x-C_{n-1}(f)(y), d y\right) \in C_{n-1}(G, \Gamma) .
$$

Now proceed as usual-noting that the chains above are dimension-wise free abelian groupsto define the (co)chain complexes with coefficients in $A$ to be:

$$
C_{*}(G, \Gamma ; A):=C_{*}(G, \Gamma) \otimes A, \quad C^{*}(G, \Gamma ; A):=\operatorname{Hom}\left(C_{*}(G, \Gamma), A\right) .
$$

This completes the definition for coefficients in any abelian group with a trivial $G$ action.

Remark: If one considers a general module $M$ over $G$, it induces a module over $\Gamma$ and one must proceed more carefully to define the appropriate relative chains and co-chains. We shall not pursue it here. Thus from now on we consider only central extensions and (co-)homology with abelian coefficients.

The map $C^{*}(f)$ together with the mapping cone gives a long exact sequences in (co)homology. In addition, from equation (2.4), one concludes that the relative homology and cohomology are related by the usual universal coefficients theorem as in $\mathrm{Sp}$, We. (Of course, there is a topological analogue, see below.)

Looking at the boundary formula of equation (2.3), ones sees that a co-cycle element in $C^{n}(G, \Gamma ; A)$ is a pair $(a, b)$, where $a \in C^{n}(G ; A)$ is a co-cycle, and $b \in C^{n-1}(\Gamma, A)$ is an explicit null cohomology of the pullback of $a$ to $C^{n}(\Gamma ; A)$.

By construction there is a sequence of three chain complexes

$$
C^{*}(G, \Gamma ; A) \rightarrow C^{*}(G ; A) \rightarrow C^{*}(\Gamma ; A) .
$$

This sequence gives, as usual for mapping cones, a long exact sequence relating the usual cohomology groups to the relative cohomology groups.

\subsection{A topological definition.}

The group cohomology of $G$ is in fact the cohomology of the associated classifying space $B G$, via the corresponding chain complexes $C_{*}(G)$. The algebraic and topological complexes are equivalent as chain complexes, hence we can take the above relative cohomology as the relative cohomology, namely $H^{*}(G, \Gamma ; A)=H^{*}(B G, B \Gamma ; A)$ and similarly for homology with coefficients in an abelian group $A$. 
Since coefficients are constant, i.e. the trivial module over the groups involved, a relative cohomology class corresponds to a homotopy class of pointed maps $[u]: B G \rightarrow K(A, 2)$, together with a class of null-homotopy $[\nu]$ of the pre-composition of $[u]$ to $B \Gamma$. This $\nu$ gives the extension of $u$ to the cone. Further $\nu$ corresponds exactly to a lift of $u \circ B(f)$ to the homotopy fibre of $u$. This lift is a map $B \Gamma \rightarrow B E$, were $E$ is the central extension of $G$ corresponding, as usual, to the algebraic cohomology class corresponding to the class $[u]$. A similar statement is true for local coefficients except that we need the usual twisted classifying space to represent a cohomology class. Proposition 2.6 below gives a detailed algebraic formulation of the topological observation above.

We now use the above basic definitions and observations in the following result, which, in details and spirit, is a direct extension of the classical classification result for central extensions of group via the second cohomology. An analogous result is true for general extensions with abelian kernel and both proofs are versions of the usual proof for the nonrelative case as in $[\mathrm{M}]$.

Proposition 2.6. The abelian group $\operatorname{Ext}^{\Gamma}(G ; A)$ of equivalence classes of central $f$-extension of $G$ with a given abelian group $A$ as the central kernel, are classified by the relative cohomology group $H^{2}(G, \Gamma ; A)$ as above.

Proof. Namely we construct a natural isomorphism:

$$
\operatorname{Ext}^{\Gamma}(G ; A) \cong H^{2}(G, \Gamma ; A)
$$

The proof is a simple extension of the classification of usual central extensions of $G$ via the second cohomology group $H^{2}(G, A)$. Let $\operatorname{Ext}^{\Gamma}(G ; A)$ be as defined in Definition 2.1.

The central extensions of $G$ with kernel $A$ are classified, up to equivalence, by the second cohomology $H^{2}(G ; A)$. Now the restriction map $f^{*}: H^{2}(G ; A) \rightarrow H^{2}(\Gamma ; A)$ takes a central extension in $H^{2}(G ; A)$ to its pull back along $f$. By Lemma 2.3 , the extensions of $G$ that are $f$-central are precisely the extensions that are sent to the trivial extension in $H^{2}(\Gamma ; A)$. This gives an exact sequence

$$
\operatorname{Ext}^{\Gamma}(G, A) \rightarrow H^{2}(G, A) \stackrel{f^{*}}{\rightarrow} H^{2}(\Gamma, A) .
$$

We show that this sequence can be extended to a 5 -term exact sequence which is the same as the usual exact sequence for $H^{2}(G, \Gamma ; A)$, namely the sequence:

$$
H^{1}(G ; A) \rightarrow H^{1}(\Gamma ; A) \rightarrow H^{2}(G, \Gamma ; A) \rightarrow H^{2}(G, A) \stackrel{f^{*}}{\rightarrow} H^{2}(\Gamma, A),
$$

but with $H^{2}(G, \Gamma ; A)$ replaced by $\operatorname{Ext}^{\Gamma}(G ; A)$.

We first define a map from $H^{1}(\Gamma, A) \cong \operatorname{Hom}(\Gamma, A)$ onto the kernel of the map $\operatorname{Ext}^{\Gamma}(G, A) \rightarrow$ $H^{2}(G, A)$. Given $\mu: \Gamma \rightarrow A$ we define an element in $\operatorname{Ext}^{\Gamma}(G ; A)$ as follows. We let $M=A \times G$, with the obvious maps $A \rightarrow A \times G \rightarrow G$, and we let $\psi: \Gamma \rightarrow M$ be defined by $\psi(\gamma)=(\mu(\gamma), f(\gamma))$, for all $\gamma \in \Gamma$. Then $(M, \psi) \in \operatorname{Ext}^{\Gamma}(G ; A)$ is in the kernel. Conversely given $(M, \psi)$ in the kernel, it is a split extension $0 \rightarrow A \stackrel{\alpha}{\rightarrow} M \rightarrow G \rightarrow 1$, with section $s: G \rightarrow M$. Thus $M=\alpha(A) \times s(G)$ and the map $\mu=\psi \circ \pi \circ \alpha^{-1}: \Gamma \rightarrow A$ defines a central $f$-extension of $G$ equivalent to $(M, \psi)$, where $\pi: M \rightarrow \alpha(A)$ is the projection. 
Finally, by Example 2.2(3), the composition map $\operatorname{Hom}(G, A) \rightarrow \operatorname{Hom}(\Gamma, A)$ takes $\operatorname{Hom}(G, A)$ by the above construction to elements in the kernel of $H^{1}(\Gamma, A) \rightarrow \operatorname{Ext}^{\Gamma}(G, A)$ by taking $\phi: G \rightarrow A$ to be the appropriate self map $((a, g) \mapsto(a-\phi(g), g)$ of the product $A \times G$.

To conclude the proof of the proposition we define an isomorphism between the exact sequence (2.5) and the exact sequence:

$$
H^{1}(G ; A) \rightarrow H^{1}(\Gamma ; A) \rightarrow \operatorname{Ext}^{\Gamma}(G ; A) \rightarrow H^{2}(G, A) \stackrel{f^{*}}{\rightarrow} H^{2}(\Gamma, A),
$$

To do that we define a natural map:

$$
\operatorname{Ext}^{\Gamma}(G ; A) \rightarrow H^{2}(G, \Gamma ; A),
$$

The five lemma then implies that it is an isomorphism.

Given an $f$-extension $(E, \psi)$ we must assign to it an element $(a, b) \in C^{2}(G, \Gamma, A)=$ $C^{1}(\Gamma ; A) \oplus C^{2}(G ; A)$. Let $[c] \in H^{2}(G ; A)$, with $c \in C^{2}(G ; A)$ a co-cycle, be the element corresponding to the extension $E$, obtained by ignoring the map $f$. Then $c$ is built as usual by choosing a set-theoretical section $s: G \rightarrow E$.

Consider now the pullback $E^{\prime}$ of $(E, \psi)$ along $f$. As we saw in Lemma 2.3, $E^{\prime}$ is a split extension of $\Gamma$ by $A$, with an explicit group theoretical section which we denoted $\psi^{\prime}$. Further, since $E^{\prime}$ is also the set theoretic pullback along $f$, the identity map $\Gamma \rightarrow \Gamma$ together with the map $f \circ s$ yields a set theoretic section $s^{\prime}: \Gamma \rightarrow E^{\prime}$. The section $s^{\prime}$ gives the co-cycle $C^{2}(f)(c)$. Of course the co-cycle given by $s^{\prime}$ is cohomologous to the co-cycle given by $\psi^{\prime}$. This gives a 1-cochain $w \in C^{1}(\Gamma, A)$ with $\partial w=C^{2}(f)(c)$, where $C^{2}(f): C^{2}(G ; A) \rightarrow C^{2}(\Gamma ; A)$ is the induced map on chains.

We have assigned the pair $(w, c) \in C^{2}(G, \Gamma ; A)$ to the $f$-central extension $E$. This construction is seen, as usual, to give a co-cycle in $C^{2}(G, \Gamma ; A)$ whose cohomology class is independent of the choice of the set theoretic section $s$. Moreover, by naturality, this definition of the map $\operatorname{Ext}^{\Gamma}(G ; A) \rightarrow H^{2}(G, \Gamma ; A)$ is compatible with the maps between the 5 terms exact sequences (2.6) and (2.5), where the other vertical maps are identity maps.

Remark 2.7. A proof similar to the proof of Proposition 2.6 shows that the proposition holds with central $f$-extensions of $G$ replaced by $f$-extensions of $G$ with a given $G$-module A.

Corollary 2.8. Assume that $f$ induces a surjection $f_{a b}: \Gamma_{a b} \rightarrow G_{a b}$, and let $A$ be an abelian group. The group of central $f$-extensions with kernel $A: \operatorname{Ext}^{\Gamma}(G ; A)$ is naturally isomorphic to $\operatorname{Hom}\left(H_{2}(G, \Gamma), A\right)$.

Proof. As we noted in subsection 2.4, the universal coefficient theorem applies in our set-up to yield the exact sequence:

$$
0 \rightarrow \operatorname{Ext}\left(H_{1}(G, \Gamma) ; A\right) \rightarrow H^{2}(G, \Gamma ; A) \rightarrow \operatorname{Hom}\left(H_{2}(G, \Gamma), A\right) \rightarrow 0 .
$$

But the short exact sequence on 1-dimensional homology: $H_{1}(\Gamma) \rightarrow H_{1}(G) \rightarrow H_{1}(G, \Gamma) \rightarrow 0$, and our surjection assumption, yields the vanishing of the first relative homology group $H_{1}(G, \Gamma)$, and thus the isomorphism. 
The construction in Definition 2.9 below, extends the Schur universal central extension of a perfect group $G$ (the case $\Gamma=1$ ), to any map between groups (not necessarily perfect groups) $f: \Gamma \rightarrow G$, inducing surjection on abelianizations.

Definition 2.9. Assume that $f$ induces a surjection $f_{a b}: \Gamma_{a b} \rightarrow G_{a b}$. We define the universal central $f$-extension of $G$ as a pair $(U, \eta) \in \operatorname{Ext}^{\Gamma}\left(G ; H_{2}(G, \Gamma)\right)$ corresponding to the identity map in $\operatorname{Hom}\left(H_{2}(G, \Gamma), H_{2}(G, \Gamma)\right.$ ) (see Corollary 2.8).

Examples: Let the map $f$ be the abelianization $f: \Gamma \rightarrow \Gamma_{a b}$. Then the universal central $f$-extension is the following with the canonical structure map $\Gamma \rightarrow \Gamma / \gamma_{3} \Gamma$ :

$$
\gamma_{2} \Gamma / \gamma_{3} \Gamma \rightarrow \Gamma / \gamma_{3} \Gamma \rightarrow \Gamma_{a b}
$$

Of course, the higher quotients give analogous examples.

If the map $f$ is a central extension then the associated universal central $f$-extension is $f$ itself with the identity as the structure map, in this case $H_{2}(G, \Gamma)$ is canonically isomorphic to the kernel of $f$. Thus for a quotient map of abelian groups $f: A \rightarrow B \cong A / K$, we recover this quotient map as the universal central $f$-extension. Given a general surjective map $f: \Gamma \rightarrow G$, the universal central $f$-extension is given by $K /[K, \Gamma] \rightarrow \Gamma /[K, \Gamma] \rightarrow G$, with the natural quotient as the structure map. Of course if $\Gamma=1$ the trivial group we recover the Schur universal central extension of the perfect group $G$.

When compared with the long exact homology sequence for a pair $G, \Gamma$, it follows that for any surjection of groups $\Gamma \rightarrow G$ with kernel $K$ we have naturally $H_{2}(G, \Gamma) \cong K /[K, \Gamma]$. The usual five terms exact sequence for a group extension follows. When applied to a generators-relations presentation of a group $G \cong F / R$ as a quotient of free group (see below) one gets as is well known, the standard version of Hopf formula for $H_{2}(G)$ as the kernel of $K /[R, F] \rightarrow \operatorname{Ker}\left(F_{a b} \rightarrow G_{a b}\right)$ which is clearly isomorphic to $R \cap[F, F] /[R, F]$ by a simple diagram chase.

To get more interesting examples one can take any group map $\Gamma \rightarrow P$, where $P$ is perfect:

2.10 A relation to extensions of perfect groups and homological obstruction to lifting solutions. In this section we consider relative extensions of a perfect group $P$. The universal $f$-extension allows one to define obstructions to the lifting of solutions of equations over $P$ to solutions over the universal Schur extension. Our discussion refers to the diagram 2.7 below.

Given a perfect group $P$, any map $f: \Gamma \rightarrow P$ is surjective on the abelian quotients, and therefore we can freely apply the results as above. Take $\Gamma=C$ a cyclic group and consider a map $f_{x}: C \rightarrow P$ determined by an element $x \in P$. Let $U_{x}$ be the universal $f_{x}$-extension. We have $H_{2}(C)=0$ and thus an exact sequence

$$
0 \rightarrow H_{2}(P) \rightarrow H_{2}(P, C) \rightarrow C \rightarrow 0 .
$$

Let $E$ be the universal Schur extension of $P$ with kernel $H_{2}(P)$. By universality of $E$ one has a map of central extension $e: E \rightarrow U_{x}$ (ignoring $f_{x}$ ). 
Let us consider the possibility of lifting the map $f_{x}: C \rightarrow P$ to $E$. For $C=C_{n}$, a cyclic group of order $n$, this would mean lifting an element of order $n$ in $P$ to an element of the same order in $E$ which, in general, is impossible.

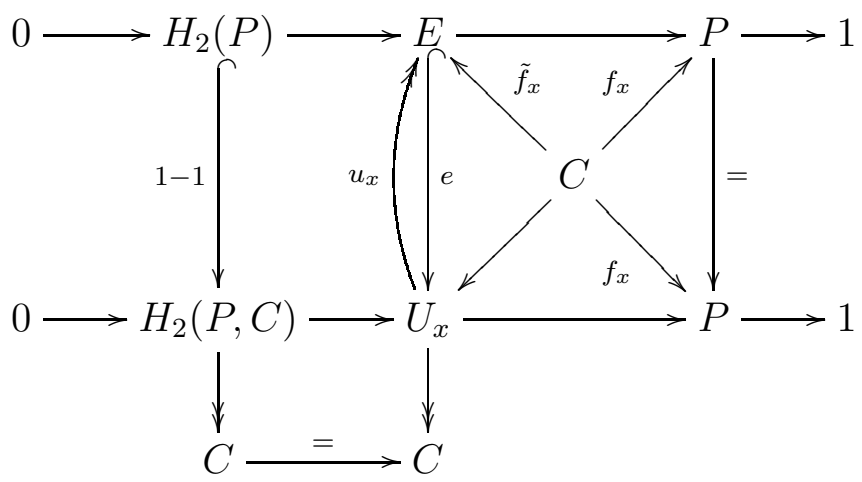

Assume that $\tilde{f}_{x}$ is a lift of $f_{x}$ to $E$. Then both extensions are equipped with a map from $\Gamma=C$ over $P$, and thus are $f_{x^{-}}$-extensions. The universality of $U_{x}$ now gives a map of $f_{x^{-}}$ extensions $u_{x}: U_{x} \rightarrow E$ left inverse to $e$. If $C=\mathbb{Z}$, the integers, a lift $\tilde{f}_{x}$ as assumed always exists. In this case the homology sequence above splits and the kernel of the universal central $f_{x}$-extension $U_{x}$ is isomorphic to $H_{2}(P) \oplus \mathbb{Z}$. Since $U_{x}$ is by itself an extension of the Schur extension $E$ of $P$, it must split by the universal properties of $U_{x}$ and $E$ hence the universal extension splits as: $U_{x} \cong E \oplus \mathbb{Z}$. The structure map $\mathbb{Z} \rightarrow E \oplus \mathbb{Z}$ corresponds to the choice $\tilde{f}_{x}$ of the lift of $x \in G$ to $E$.

When $C$ is a finite cyclic group, the sequence $(\star)$ above does not split in general but under our assumption of the existence of a lift $\tilde{f}_{x}$ we conclude that $U_{x} \rightarrow E$ has a section and thus $(\star)$ does split. We conclude that the splitting of $(\star)$ is a necessary condition for the existence of a lift $f_{x}$. In other words the extension sequence $(\star)$ is an obstruction element in $\operatorname{Ext}\left(C, H_{2}(P)\right)$ to the lifting problem of $x \in P$.

Similarly, for any map $f: \Gamma \rightarrow P$, and let $U$ be the universal central $f$-extension. Assume that $f$ lifts to $\tilde{f}: \Gamma \rightarrow E$, then, one must have a splitting of $U \rightarrow E$ as argued above, since both $E$ and $U$ have their initial-universal properties. Note that the canonical map $e: E \rightarrow U$ is not a map of central $f$-extension while $u: U \rightarrow E$ is such a map, thus we have $e \circ u=I d_{E}$ but in general $u \circ e$ is not the identity on $U$.

Looking at the kernels of the extensions one gets that $\mathrm{H}_{2} \mathrm{\Gamma} \rightarrow \mathrm{H}_{2} \mathrm{P}$ is the zero map.

Consider for example a map $f: \Gamma=\mathbb{Z} \oplus \mathbb{Z} \rightarrow P$ given by a choice of two commuting elements $x, y \in P$. This map, in general, cannot be lifted to the Schur extension $E$ of $P$. We look for a necessary condition for such a lift to exists, namely for an obstruction for lifting this pair of commuting elements in $P$ to commuting elements in $E$. Assume we have a lift of $f$ i.e. $\tilde{f}: \mathbb{Z} \oplus \mathbb{Z} \rightarrow E$. 
A lift gives a map $U \rightarrow E$ by universality of $U$, thus getting a split short exact sequence, since $E \rightarrow U$ always exists by the universality of $E$ :

$$
1 \rightarrow \mathbb{Z} \oplus \mathbb{Z} \rightarrow U \rightarrow E \rightarrow 1
$$

This means that the natural map $H_{2}(\Gamma)=H_{2}(\mathbb{Z} \oplus \mathbb{Z})=\mathbb{Z} \rightarrow H_{2}(P)$ induced by the map $f$ must be zero, as argued above. Hence the relevant element in the second homology is an obstruction to the lift: The injection $E \rightarrow U$ induces a injection on the kernels of the extensions: $H_{2}(P) \hookrightarrow H_{2}(P, \mathbb{Z} \oplus \mathbb{Z})$.

More generally, starting with elements $x_{i} \in G$ satisfying equations $w_{a}\left(x_{1}, x_{2} \ldots\right)=1$ one gets a homological obstruction for lifting $x_{i}$ to elements $e_{i} \in E$ satisfying the same conditions $w_{a}\left(e_{i}\right)=1$ taking $\Gamma=$ Free $/ w_{a}$ namely the vanishing of $H_{2}(\Gamma) \rightarrow H_{2}(P)$, where $H_{2}(\Gamma)$ can be given explicitly in terms of these relations.

\subsection{The universality of $U$.}

We now turn to the universal $f$-extension $U-$ a relative version of the universality of the Schur central extension of perfect groups.

Theorem 2.12. Assume that the map $f_{a b}: \Gamma_{a b} \rightarrow G_{a b}$ induced on the abelianizations is surjective, and let $(U, \eta)$ be the universal central $f$-extension of Definition 2.9. Then for any central $f$-extension $(E, \psi)$ of $G$ there is a unique map of central $f$-extensions (as in Definition 1.1(2)) from $U$ to $E$. The map on the abelianization $\Gamma_{a b} \rightarrow U_{a b}$ is surjective.

Proof. By Corollary 2.8 , there is a $1-1$ correspondence between central $f$-extensions of $G$ with kernel $A$ and $\operatorname{Hom}\left(H_{2}(G, \Gamma), A\right)$. Furthermore

Lemma 2.13. The isomorphism of Corollary 2.8 can be built for the universal $f$-extension $(U, \eta)$ by taking $e \in \operatorname{Hom}\left(H_{2}(G, \Gamma), A\right)$ to the element $(E, \psi)$ in $\operatorname{Ext}^{\Gamma}(G ; A)$ as in the following pushout diagram, where $E$ is the pushout of $\iota$ and $e$, and $\psi=\eta \circ \tau$.

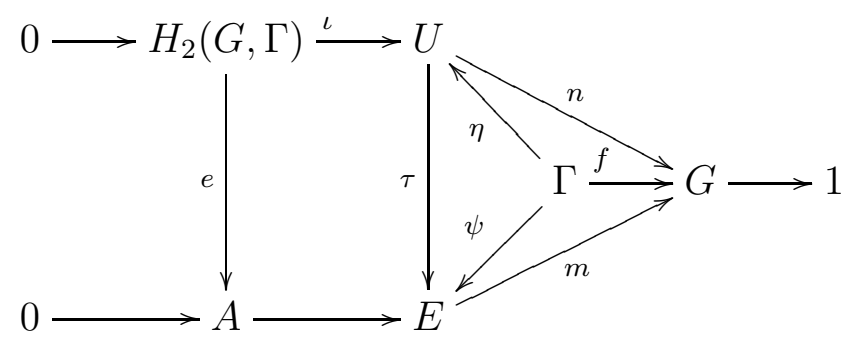

Proof. By naturality, given any map $a \in \operatorname{Hom}\left(H_{2}(G, \Gamma), A\right)$, the following diagram commutes

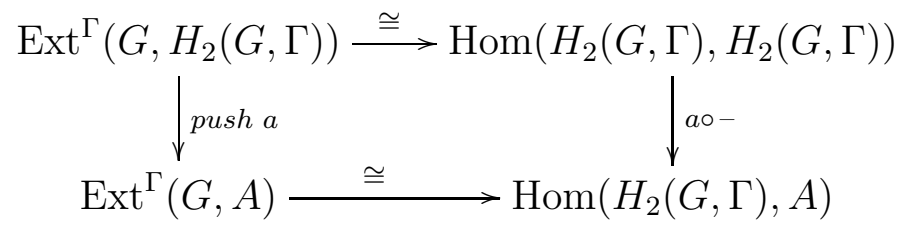


where the map "push $a$ " is the map obtained by a pushout diagram as in diagram (2.8) above, with $(U, \eta)$ replaced by some element in $\operatorname{Ext}^{\Gamma}\left(G, H_{2}(G, \Gamma)\right)$, and where $e$ is replaced by $a$. The map $a \circ-$ is composition with $a$.

Let $(M, \psi) \in \operatorname{Ext}^{\Gamma}(G, A)$. It corresponds uniquely to $a \in \operatorname{Hom}\left(H_{2}(G, \Gamma), A\right)$. Now consider diagram (2.9) with that map $a$. Then $a=a \circ \mathrm{id}_{H_{2}(G, \Gamma)}$, i.e, $a$ comes from the identity in $\operatorname{Hom}\left(H_{2}(G, \Gamma), H_{2}(G, \Gamma)\right)$, and we see that $(M, \psi)$ occurs as a pushout of $(U, \eta)$.

Lemma 2.13 shows that each $f$-central extension $(E, \psi)$ of $G$ with kernel $A$ occurs uniquely as a pushout of $e \in \operatorname{Hom}\left(H_{2}(G, \Gamma), A\right)$. The desired map $U \rightarrow E$ is the map $\tau$ of $\operatorname{diagram}$ (2.8).

Remark 2.14. The results of this section were used in [FS, Theorem 5.4] to identify the kernel of the free normal closure $\Gamma^{f}$, in the case where $G=\left\langle f(\Gamma)^{G}\right\rangle$.

In the above paragraphs we have proved the main part of theorem 2.12 . To conclude the proof of Theorem 2.12 we consider a basic property of the universal extension that extends a familiar property of the universal central extension of a perfect group:

Lemma 2.15. If $f$ induces a surjection on abelianization then the same is true for the universal extension $(U, \eta)$ : the associated structure map of the extension $\eta: \Gamma \rightarrow U$ is also surjective on the abelian quotients.

Proof. Here we provide two proofs, one direct and one using a 5-term exact sequence.

For a direct proof, first note that $\eta_{a b}$ is surjective if and only if $\eta \mapsto \eta \circ \mu$ is an injective $\operatorname{map} \operatorname{Hom}(U, A) \rightarrow \operatorname{Hom}(\Gamma, A)$, for any abelian group $A$. Now given a map $a: U \rightarrow A$ to any abelian group whose pre-composition to $\Gamma$ is zero, we show that $a=0$ : Consider the trivial $f$-extension of $G$ namely $A \times G$; where this trivial extension is equipped with the structure $\operatorname{map} 0 \times f: \Gamma \rightarrow A \times G$.

Let $u: U \rightarrow G$ be the unique map of Theorem 2.12. Now construct two maps between these $f$-extensions $(U, \eta)$ and $(A \times G, 0 \times \eta)$, namely the maps $a \times u, 0 \times u: U \rightarrow A \times G$.

The condition on the pre-composition implies that these two maps are maps of $f$-extensions as defined above, since both are $0 \times f$ on $\Gamma$, commutativity on $\Gamma$ follows.

Thus both are well defined maps of factorizations and therefore by uniqueness of maps of factorization from the universal $(U, \eta)$, they are equal and we get $a=0$ as needed.

Here is a second proof using a relative 5-terms exact sequence. Start with any central $f$-extension of the form $A \rightarrow E \rightarrow G$ with $\psi: \Gamma \rightarrow E$ as the structure map. We have the exactness of the following relative version of the usual 5-term sequence.

$$
H_{2}(E, \Gamma) \rightarrow H_{2}(G, \Gamma) \stackrel{\partial}{\rightarrow} A \rightarrow H_{1}(E, \Gamma) \rightarrow H_{1}(G, \Gamma) \rightarrow 0 .
$$

To see this one can proceed algebraically by chasing the relevant diagram of exact sequences, but here is a topological argument:

Comparing the exact sequence of the cofibration $B E \rightarrow B G \rightarrow B G / B E$ with the usual 5 -term exact sequence of lower homology groups (i.e. with $\Gamma=1$ in $(\star \star)$ above) implies (by the 5-lemma) the isomorphism $H_{2}(B G / B E) \cong A$. 
Therefore the homology exact sequence, in low dimensions, of the cofibration sequence involving three mapping cones:

$$
B E / B \Gamma \rightarrow B G / B \Gamma \rightarrow B G / B E,
$$

yields the desired 5 -terms exact sequence in homology.

Now consider the 5 -terms exact sequence in relative homology associated to the universal extension sequence:

$$
0 \rightarrow H_{2}(G, \Gamma)=A \rightarrow U \rightarrow G \rightarrow 1
$$

of the universal central $f$-extension with the structure map $\Gamma \rightarrow U$ :

We note that the connecting boundary map $\partial$ in the low homology sequence is an isomorphism by construction. We have by assumption that $H_{1}(G, \Gamma) \cong 0$, we get immediately for the exact sequence the vanishing $H_{1}(U, \Gamma) \cong 0$, and thus surjectivity on the first homology as needed.

\section{UNIVERSAL FACTORIZATIONS}

Next we consider the universal properties of $U_{\infty}$, as stated in theorem 1.5 of the introduction: Characterizing the factorization $\Gamma \rightarrow U_{\infty} \rightarrow G$ as both initial and terminal.

3.1 Relative Schur Tower. In light of Lemma 2.15, we can repeat the construction of the universal central $f$-extension. We get a tower

$$
\Gamma \rightarrow U_{\infty} \rightarrow \cdots U_{n+1} \rightarrow U_{n} \rightarrow \cdots U_{1}=U \rightarrow U_{0}=G
$$

As we will presently see, for finite groups, it stop after a finite number of steps at the universal hypercentral extension of $G$.

Proposition 3.2. The above tower stops: For any map $f: \Gamma \rightarrow G$ of finite groups as above, with $f_{a b}$ surjective, the inverse limit $U_{\infty}$ is a finite group which is equal to $U_{n}$ for all sufficiently large $n$.

To prove that the tower stops we use a slight generalization of the argument given in Lemma 4.4 and Lemma 4.5 of [FS]. The same proof goes through using the following slight modification which is a basic observation on nilpotent groups

Lemma 3.3. Let $g: G \rightarrow N$ be a map with $N$ being nilpotent, such that $g$ induces a surjection on abelianization then $g$ is surjective. In particular, if $g$ is also injective then $G=N$.

Proof. The only property of the nilpotent group $N$ used here is that its Frattini quotient $N / \Phi(N)$ is an abelian group. We can assume $G$ is a subgroup of $N$ by looking at its image under $f$. The assumption of surjection $G \rightarrow N_{a b}$ implies that $G$ together with the commutator subgroup of $N$ generate $N$, i.e. $N=[N, N] G$. Since $[N, N] \leq \Phi(N)$, we get $G \Phi(N)=N$. But by the basic property of $\Phi(N)$ this means $G=N$. 
Remark: Topologically the relative Schur tower is gotten by repeatedly taking the homotopy fibre of the composition:

$$
B G \rightarrow B G / B \Gamma \rightarrow P_{2}(B G / B \Gamma)
$$

as a sort of "relative modified Bousfiled-Kan homology completion tower". Moreover, this last construction is well defined without any assumption of $\Gamma \rightarrow G$.

Proposition 3.4. Let $f: \Gamma \rightarrow G$ be a map of finite groups, surjective on abelian quotients. The limit $U_{\infty}$ of the tower $U_{i}$ of repeated universal central $f$-extensions is terminal among all factorizations of $\Gamma \rightarrow E \rightarrow G$ of $f$ which are low-dimensional-acyclic factorization namely with $H_{i}(E, \Gamma)=0$ for $i=1,2$.

The initial extension here is the trivial $\Gamma \rightarrow M=\Gamma \rightarrow G$.

Dually one has

Proposition 3.5. Let $f: \Gamma \rightarrow G$ be a map of finite groups, surjective on abelian quotients. The limit $U_{\infty}$ of the tower $U_{i}$ of repeated universal central $f$-extensions is initial among all hypercentral factorization $f: \Gamma \rightarrow M \rightarrow G$ of maps $f: \Gamma \rightarrow G$. In addition it is lower acyclic namely, $H_{i}\left(U_{\infty}, \Gamma\right)=0$ for $i=1,2$.

The terminal hypercentral extension is of course $\Gamma \rightarrow M=G \rightarrow G$.

Proofs of universality. To prove the initial property of $U_{\infty}$ we use of course that $U=U_{1}$ itself is initial among all central $f$-extensions. Now we proceed by induction. Given any hypercentral extension of $G$ it comes with a finite tower $E_{n} \rightarrow E_{n-1} \rightarrow \cdots \rightarrow E_{1} \rightarrow G$, where $E_{i+1} \rightarrow E_{i}$ etc. is a central $f$-extension. Now we assume we have a map $U_{\infty}=U_{N}$ to $E_{i}$. then by the universality of $U$ we also have a map $U_{N+1} \rightarrow E_{i+1}$. But we know that $U_{N+1}=U_{N}=U_{\infty}$. Hence we get a map of hypercentral $f$-extensions. Uniqueness follows similarly, compare with [FS].

We turn to the terminal property of $U_{\infty}$. Let $\Gamma \rightarrow M \rightarrow G$ be a factorization by some $f$ extension with $H_{i}(M, \Gamma)=0$ for $i=1,2$. Consider the universal factorization $\Gamma \rightarrow W_{\infty} \rightarrow M$ of $\Gamma \rightarrow M$. It maps to $U_{\infty}$. The map $M \rightarrow G$ induces by naturality a map $W_{\infty} \rightarrow U$. But we saw above that the kernel of $W \rightarrow M$ is $H_{2}(M, \gamma)=0$ by assumption on $M$. Therefore $W=M$ we get a map $M \rightarrow U$. repeating this we get a map $M \rightarrow U_{\infty}$. Uniqueness follows by a similar argument using $H_{2}\left(U_{\infty}, \Gamma\right)=0$.

\section{A remark on a relation between $U_{\infty}$ and $\Gamma_{\infty}$ :}

Together with the $\Gamma_{\infty}$ factorization of [FS] we have two universal constructions for maps of finite groups: One, $\Gamma \rightarrow \Gamma_{\infty} \rightarrow G$, is initial among all subnormal factorizations i.e. $\Gamma \rightarrow S \rightarrow G$ with $S \rightarrow G$ a subnormal map; the other, $\Gamma \rightarrow U_{\infty} \rightarrow G$ is terminal among all factorization with $H_{i \leq 2}(-, \Gamma)=0$. Both have trivial relative homology groups $H_{* \leq 2}(-, \Gamma) \cong 0$. Note that in the second construction above, $U_{\infty}$, it is assumed that the map $\Gamma \rightarrow G$ is surjective on the abelian quotients.

However, one may well start with an arbitrary map $f: \Gamma \rightarrow G$ and proceeds by first taking the maximal normal subgroups $B \subseteq G$ containing the image of $f$ for which the map 
$f_{B}: \Gamma \rightarrow B$ is surjective on the abelian quotient. such a maximal subgroup exists for any map in a natural way. In this way the universal $f_{B}$ hypercentral extension $\Gamma \rightarrow U_{B} \rightarrow B$ gives a subnormal map $U_{B} \rightarrow G$ factorizing $f$ in the desired way. Thus both $\Gamma_{\infty}$ and $U_{\infty}:=U_{B}$ (by definition for an arbitrary map) are defined for arbitrary maps of finite groups and both give subnormal factorizations in the sense of [FS]; and now again $\Gamma_{\infty}$ is initial and $U_{\infty}$ as defined here is terminal. Since $U_{\infty}$ is subnormal by construction being a hypercentral extension of a normal subgroup $B \unlhd G$, there is a unique natural map $\Gamma_{\infty} \rightarrow U_{\infty}$ commuting with the factorization diagrams. Dually this map exists because of the initial property of $U_{\infty}$ as above. For example, if we take $G=P$ a perfect group and $\Gamma=1$ the trivial group then $\Gamma_{\infty}=1$ and $U_{\infty}=E$ the universal Schur extension of $P$.

\section{REFERENCES}

[E] G. Ellis, The Schur multiplier of a pair of groups, Appl. Categ. Structures 6 (1998), no. 3, 355-371.

[FS] E. D. Farjoun, Y. Segev, Normal closure and injective normalizer of a group homomorphism, submitted, 2014.

[H] G. Hochschild, Relative homological Algebra, Trans. Amer. Math. Soc., Vol. 82, No. 1, 1956 pp. 246-269.

[Ka] G. Karpilovsky, The Schur multiplier, London Math. Soc. Monogr. bf 2, Oxford University Press, Oxford 1987.

[M] S. MacLane, Homology, Reprint of the 1975 edition. Classics in Mathematics. Springer-Verlag, Berlin, 1995.

[Sp] E. H. Spanier, Algebraic topology, Corrected reprint. Springer-Verlag, New York-Berlin, 1981.

[We] C. A. Weibel, An introduction to homological algebra, Cambridge Studies in Advanced Mathematics, 38. Cambridge University Press, Cambridge, 1994.

Emmanuel D. Farjoun, Department of Mathematics, Hebrew University of Jerusalem, Givat Ram, Jerusalem 91904, IsRael

E-mail address: farjoun@math.huji.ac.il

Yoav Segev, Department of Mathematics, Ben Gurion University, Beer Sheva 84105, IsRAEL

E-mail address: yoavs@math.bgu.ac.il 